$56^{\text {ème }}$ Congrès de la SFMBCB, 03013 (2011)

DOI: $10.1051 / \mathrm{sfmbcb} / 20115603013$

(C) Owned by the authors, published by EDP Sciences, 2011

\title{
Le pemphigus vulgaire : présentation d'une observation
}

\author{
Chbicheb S, El Wady W \\ Service d'Odontologie chirurgicale, CCTD, Rabat, Maroc \\ s_chbicheb@yahoo.fr
}

Le pemphigus vulgaire est une dermatose bulleuse auto-immune. Il représente la forme la plus fréquente (85 \% des cas de pemphigus). Considéré comme une maladie de l'adulte puisque débutant le plus souvent entre 40 et 60 ans, il peut néanmoins être observé chez l'adolescent et le nouveau-né. Sa prédominance féminine est encore discutée (Mimouni 2002).

Sur le plan pathogénique, cette dermatose bulleuse constitue un modèle physiopathologique de maladie auto-immune. Elle résulte non seulement de la destruction du système d'adhésion par des autoanticorps, mais aussi de l'altération de la fonction d'adhésion des protéines, due à la fixation des autoanticorps sur leur cible antigénique. Ceci aboutit formation des bulles caractéristiques de différents pemphigus auto-immuns (Bédane 2003).

Un patient âgé de 41 ans a consulté au Service d'Odontologie chirurgicale du CCTD de Rabat pour des lésions érosives de la muqueuse jugale, linguale et labiale, évoluant depuis un mois. L'interrogatoire du patient révèle une gêne douloureuse au moment de l'alimentation. Aucune prise médicamenteuse durant les derniers mois n’a été rapportée. Les lésions buccales sont apparues progressivement, sous forme de bulles isolées qui se sont rapidement rompues, laissant place à des érosions confluentes, prenant l'aspect de plages érythémateuses. L'examen exobuccal montre des lésions croûteuses sur le vermillon. L'examen endobuccal a mis en évidence une atteinte buccale généralisée.

Le frottis réalisé sur la muqueuse jugale qui a montré une acantholyse, Les examens cytologique et histologique, complétés par une immunofluorescence directe, ont conclu à un pemphigus vulgaire. Le patient, adressé à un dermatologue, a bénéficié d'un traitement à base de corticoïdes par voie générale, à raison de $2 \mathrm{mg} \mathrm{Kg}^{-1} \cdot \mathrm{j}^{-1}$. Le patient a été revu un mois après pour l'éradication des foyers infectieux buccodentaires. Des extractions dentaires ont été réalisées sous antibiothérapie, en raison du risque infectieux que représente l'immunosuppression induite par la corticothérapie.

Cliniquement, le pemphigus vulgaire débute dans les 2/3 des cas par des lésions buccales précédant de 2 à 3 mois les lésions cutanées. Dans certains cas, les lésions buccales du pemphigus restent isolées (Claudy 2001), ce qui correspond à notre cas. A l'inverse, environ $15 \%$ des pemphigus vulgaires n'ont jamais de lésions buccales (Vaillant 1999). Ces lésions peuvent se distribuer à toute la cavité buccale, mais prédominent habituellement aux zones de frottement : face interne des joues, palais (70 à $78 \%$ ) et gencive attachée (20 à $25 \%$ des cas) (Bystrun 2002). L'atteinte cutanée, lorsqu'elle survient, apparaît secondairement et se traduit par des bulles flaccides siégeant en peau saine, rapidement remplacées par des érosions postbulleuses avec collerette épidermique. Le signe de Nikolsky est positif en peau péribulleuse. Le diagnostic repose sur l'examen histologique (bulle intra-épithéliale contenant des cellules acantholytiques) et la présence d'anticorps en immunofluorescence directe (dépôts intercellulaires dans l'épithélium donnant un aspect en résille). Le traitement est basé sur la corticothérapie par voie générale à doses faibles, associée à des immunosuppresseurs, en cas d'atteinte uniquement buccale. Une atteinte cutanée associée, nécessite le recours à une corticothérapie générale à fortes doses (Dereure 2002). 